\title{
Numerical Investigation of Tungsten Reduction Process
}

\author{
Natalia Gicheva ${ }^{1}$, Alexander Shvab ${ }^{1}$, and Vladimir Brendakov, ${ }^{2, *}$ \\ ${ }^{1}$ National Research Tomsk State University, 634050 Tomsk, Russia \\ ${ }^{2}$ Seversk Technological Institute, 636036 Seversk, Russia
}

\begin{abstract}
The subject of this research was a chemical reactor for the producing tungsten coverings by means of chemical vapor deposition. The physical and mathematical model of viscous incompressible fluid motion and heat and mass transfer in a vortex chamber has been described. The velocity field, the temperature and the concentration distributions in a vortex chamber were obtained. Calculations reliability was verified by different methods. Parametric studies on effects of the Reynolds, Prandtl and Rossbi criteria on the flow characteristics were performed.
\end{abstract}

\section{General mathematical model}

\subsection{System of equations}

In this paper the process of tungsten deposition from the tungsten hexafluoride and hydrogen mixture is considered. The stoichiometry of that is:

$$
\mathrm{WF}_{6}+3 \mathrm{H}_{2}=\mathrm{W}+6 \mathrm{HF} \text {. }
$$

The numerical simulation of hydrodynamics and heat and mass transport for viscous compressible gas in a cylindrical reactor is carried out in this work (Fig. 1) [1]. The gas having axial velocity $U_{0}$, temperature $T_{0}$ and concentration $C_{0}$ enters from the pipe in the center of chamber, and then exits through the annual channel on the periphery of that. The top and the bottom of a vortex chamber are rotating with a constant angular velocity $\omega$. A susceptor with a temperature $T_{l}$ fills a part of the bottom, the tungsten reduced deposits there.

In this model a binary mixture is considered, the first component of one is tungsten hexafluoride with hydrogen and the second component is hydrogen fluoride. By virtue of low velocities and temperature differences the fluid considered is incompressible, but density depends on concentration because component ratio changes during the reaction. The density of a mixture is determined by the additive principle by means of the mass concentrations and the true densities of the corresponding components.

\footnotetext{
* Corresponding author: vnbrendakov@mephi.ru
} 


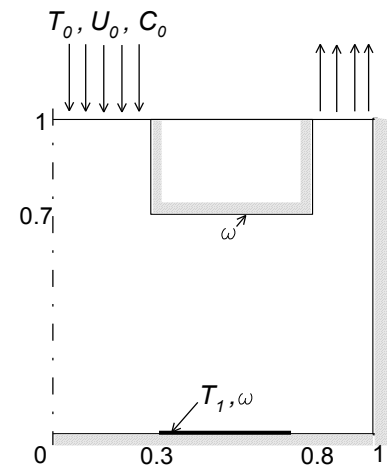

Fig. 1. A schematic image of a vortex chamber of reactor.

The system of equations describing processes in a vortex chamber contains viscous incompressible fluid dynamics (1-3), mass conservation (4), energy (5) and mass transport (6) equations. It can be represented in dimensionless form in a cylindrical coordinates:

$$
\begin{aligned}
& \frac{\partial\left(\rho u_{r}\right)}{\partial t}+\frac{\partial\left(\rho u_{r}\right)^{2}}{\partial r}+\frac{\partial\left(\rho u_{z} u_{r}\right)}{\partial z}=-\frac{\partial p}{\partial r}-\frac{\rho u_{r}^{2}}{r}+\frac{\rho u_{\varphi}^{2}}{r}+ \\
& +\frac{2}{\operatorname{Re}} \frac{\partial}{\partial r}\left(\mu \frac{\partial u_{r}}{\partial r}\right)+\frac{1}{\operatorname{Re}} \frac{\partial}{\partial z}\left[\mu\left(\frac{\partial u_{r}}{\partial z}+\frac{\partial u_{z}}{\partial r}\right)\right]+\frac{1}{r} \frac{1}{\operatorname{Re}}\left(2 \mu \frac{\partial u_{r}}{\partial r}-2 \mu \frac{u_{r}}{r}\right) \\
& \frac{\partial\left(\rho u_{z}\right)}{\partial t}+\frac{\partial\left(\rho u_{r} u_{z}\right)}{\partial r}+\frac{\partial\left(\rho u_{z}\right)^{2}}{\partial z}=-\frac{\partial p}{\partial z}-\frac{\rho u_{r} u_{z}}{r}+ \\
& +\frac{1}{\operatorname{Re}} \frac{\partial}{\partial r}\left[\mu\left(\frac{\partial u_{z}}{\partial r}+\frac{\partial u_{r}}{\partial z}\right)\right]+\frac{2}{\operatorname{Re}} \frac{\partial}{\partial z}\left(\mu \frac{\partial u_{z}}{\partial z}\right)+\frac{1}{\operatorname{Re}} \frac{\mu}{r}\left(\frac{\partial u_{z}}{\partial r}+\frac{\partial u_{r}}{\partial z}\right)+\frac{G r}{\operatorname{Re}^{2}} \theta \\
& \frac{\partial\left(\rho u_{\varphi}\right)}{\partial t}+\frac{\partial\left(\rho u_{r} u_{\varphi}\right)}{\partial r}+\frac{\partial\left(\rho u_{z} u_{\varphi}\right)}{\partial z}=-\frac{2 \rho u_{r} u_{\varphi}}{r}+ \\
& +\frac{1}{\operatorname{Re}} \frac{\partial}{\partial r}\left[\mu\left(\frac{\partial u_{\varphi}}{\partial r}-\frac{u_{\varphi}}{r}\right)\right]+\frac{1}{\operatorname{Re}} \frac{\partial}{\partial z}\left(\mu \frac{\partial u_{\varphi}}{\partial z}\right)+\frac{1}{\operatorname{Re}} \frac{2 \mu}{r}\left(\frac{\partial u_{\varphi}}{\partial r}-\frac{u_{\varphi}}{r}\right) \\
& \frac{\partial \rho}{\partial t}+\frac{\partial\left(\rho u_{r}\right)}{\partial r}+\frac{\partial\left(\rho u_{z}\right)}{\partial z}+\frac{\rho u_{r}}{r}=0 ; \\
& \frac{\partial \rho \theta}{\partial t}+\frac{\partial\left(\rho u_{r} \theta\right)}{\partial r}+\frac{\left(\partial \rho u_{z} \theta\right)}{\partial z}=\frac{1}{\operatorname{Re} \cdot \operatorname{Pr}}\left(\frac{\partial^{2} \theta}{\partial r^{2}}+\frac{1}{r} \frac{\partial \theta}{\partial r}+\frac{\partial^{2} \theta}{\partial z^{2}}\right)+ \\
& +\frac{\left(1-k_{c}\right)}{\operatorname{Re}_{D} \operatorname{Pr}_{D}}\left[\frac{1}{r} \frac{\partial}{\partial r}\left(\theta \rho r \frac{\partial C}{\partial r}\right)+\frac{\partial}{\partial z}\left(\theta \rho \frac{\partial C}{\partial z}\right)\right]-\frac{\rho u_{r} \theta}{r} \\
& \frac{\partial(\rho C)}{\partial t}+\frac{\partial\left(\rho u_{r} C\right)}{\partial r}+\frac{\left(\partial \rho u_{z} C\right)}{\partial z}=\frac{1}{\operatorname{Re} \cdot \operatorname{Pr}_{D}}\left[\frac{\partial}{\partial r}\left(\rho \frac{\partial C}{\partial r}\right)+\frac{\rho}{r} \frac{\partial C}{\partial r}+\frac{\partial}{\partial z}\left(\rho \frac{\partial C}{\partial z}\right)\right]-\frac{\rho u_{r} C}{r}
\end{aligned}
$$


For non-dimensional system (1-6) to obtain the following parameters were used: radius of the vortex chamber, linear velocity at the input, density and viscosity of a gas mixture at the input and maximal temperature difference. For a numerical solution of the momentum transport equations the time-splitting method and the method of pressure and velocity correction were used. Transport equations are solved by dint of the alternating direction implicit scheme. The solving is realized on a staggered grid.

\subsection{Boundary conditions}

For a unique solution to obtain, the boundary conditions in dimensionless form are set. At the input of chamber we have:

$$
u_{r}=0, \quad u_{z}=-1, \quad u_{\varphi}=0, \quad \theta=0, \quad C=1 ;
$$

at the symmetry axis by $r=0$ we have:

$$
u_{r}=0, \quad \frac{\partial u_{z}}{\partial r}=0, \quad u_{\varphi}=0, \quad \frac{\partial \theta}{\partial r}=0, \quad \frac{\partial C}{\partial r}=0
$$

at the bottom on a susceptor:

$$
u_{r}=0, \quad u_{z}=0, \quad u_{\varphi}=R \omega \cdot r, \quad \theta=1, \quad \frac{\partial C}{\partial z}=k C^{n} .
$$

The reaction rate constant $k$ basically depends on a susceptor temperature and size of particles deposited. Outside the susceptor and at the top wall of chamber:

$$
u_{r}=0, \quad u_{z}=0, \quad u_{\varphi}=R \omega \cdot r, \quad \frac{\partial \theta}{\partial z}=0, \quad \frac{\partial C}{\partial z}=0 ;
$$

at the right wall and at the walls of input and output channels ( $n$ is normal direction):

$$
u_{r}=0, \quad u_{z}=0, \quad u_{\varphi}=0, \quad \frac{\partial \theta}{\partial n}=0, \quad \frac{\partial C}{\partial n}=0
$$

at the chamber output, the Neumann conditions are used for all functions: $\partial / \partial z=0$.

\section{Results and discussion}

As a result of a numerical solution the distributions of velocity, temperature and concentrations were obtained. Influence of flow velocity, natural convection and rotation degree is studied.

An analysis performed let conclude that the parameters of a gas flow significant effect on its behavior and therefore on the matter layer depositing on a susceptor. This let regulate the reactor operating conditions for the optimal result to obtain. So, a rotation leads to a more uniform distribution of a temperature field and a matter near the susceptor. This occurs due to a uniform thickness boundary layer generation. At the figure 2 the streamlines $(a)$, the temperature field $(b)$ and the concentration contour lines $(c)$ in a reactor chamber are represented. The flow parameters: $\operatorname{Re}=50, \operatorname{R} \omega=5, \mathrm{Gr}=10^{4}, \operatorname{Pr}=1, \operatorname{Pr}_{\mathrm{D}}=1$. 

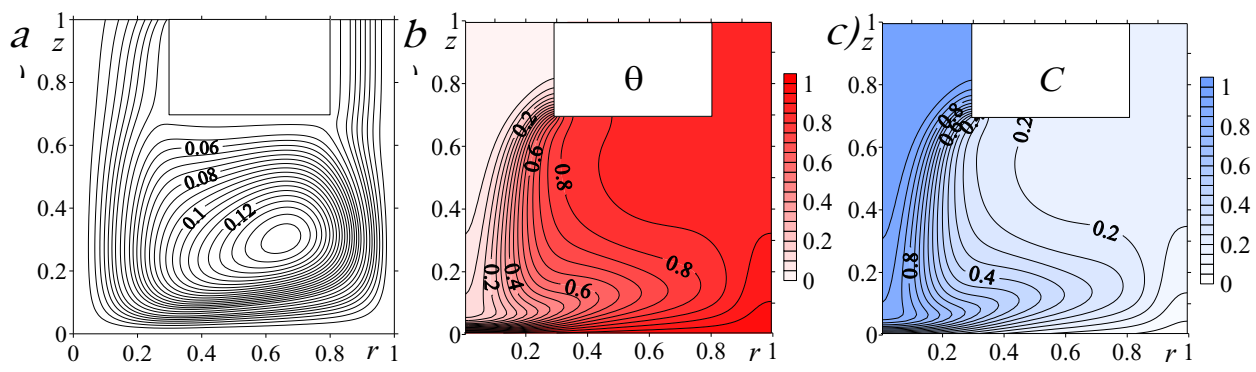

Fig. 2. The streamlines, temperature and concentration distributions in a vortex chamber depending on rotation availability.

\section{Reliability proof}

Verification of model created was performed by different methods: comparison of two solving methods, comparison with analytical solution for annual channel by low Reynolds number, test problem solution and qualitatively comparison with results known in literature.

The flow due to a rotating disk was considered. The model developed was applied to this problem solving. Fig. 3 shows a comparison between the calculation results and Cochran's exact solution. Dots denote exact values; solid lines represent the model results.

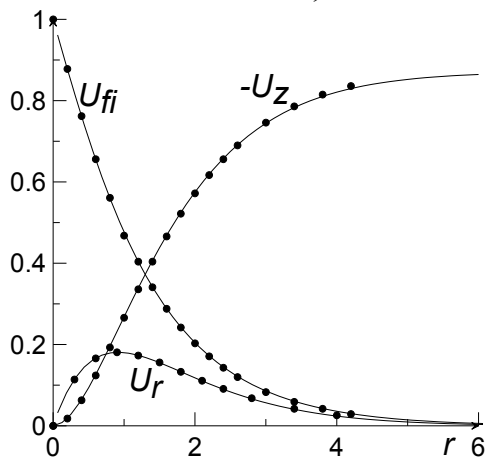

Fig. 3. Velocities distribution near a rotating disk.

\section{Conclusions}

The physical and mathematical model of viscous incompressible fluid motion and heat and mass transfer in a vortex chamber has been described. Parametric studies were performed. A significant influence of a natural convection and flow rotation was found. The study performed allows selecting reactor operating conditions for the optimal result and uniform tungsten covering to obtain.

\section{References}

1. V. N. Brendakov, A. A. Demidenko, A. V. Shvab, N. S. Evseev, The method for obtaining a tungsten ware by layer-by-layer deposition of tungsten and a device for its implementation, Pat. No. 2641596 (2018) [in Russian] 